\title{
Atributos físicos e químicos de solos de pomares de laranja doce no semiárido do Ceará, Brasil
}

\author{
Kassio Ewerton Santos Sombra ${ }^{1}$, Alexandre Caique Costa e Silva ${ }^{2}$, \\ Francisco Leandro Costa Loureiro ${ }^{3}$, José Alex do Nascimento Silva ${ }^{4}$, \\ Luiz Gonzaga dos Santos Filho ${ }^{5}$ \& Cleilson do Nascimento Uchôa ${ }^{2}$
}

\section{RESUMO}

A citricultura tem se desenvolvido em diversas classes de solos, até em solos de baixa fertilidade natural, conhecendo previamente suas características físico-químicas para inferir o manejo adequado, sejam métodos de correção e adubação, ou práticas conservacionistas que minimizem a degradação e a erosão dos solos agricultáveis, o que tende a agravar-se no clima quente e seco. Diante disto, o trabalho avaliou e comparou os atributos físicos e químicos dos solos de pomares de laranja de Russas (Citrus sinensis L. Osbeck) (Rutaceae), com plantas em estágio produtivo ou juvenil, na agricultura familiar do semiárido cearense. As características físicas e químicas avaliadas consistiram da composição granulométrica, densidade das partículas e do solo, determinando-se a classe textural, além dos teores de carbono orgânico e matéria orgânica; dos macros e micronutrientes, como por exemplo, fósforo e potássio disponíveis, ou teores de sódio, cálcio, magnésio e alumínio trocáveis; assim como, micronutrientes essenciais. Determinando-se a soma das bases, a capacidade de troca de cátions, saturação por bases trocáveis e a saturação por sódio. Os solos dos pomares produtivos e juvenis não diferiram significativamente, e apresentavam, predominantemente, textura 'franca', com baixos teores de carbono e matéria orgânica, além de alcalinidade fraca e teores satisfatórios dos macro e micronutrientes essenciais, apresentando valores elevados para soma das bases, capacidade de troca catiônica e saturação por bases, necessitando de correções e adubações, assim como, uso de práticas conservacionistas que contribuam para melhoria da qualidade do solo, e consequentemente, obtenção de maiores produtividades nos pomares cítricos.

Termos de indexação: citros, fertilidade, laranja de Russas, manejo.

Physical and chemical attributes of sweet orange orchard soils in the semi-arid of ceará, Brazil

\section{SUMMARY}

Citriculture has been developed in several soil classes, even in soils with low natural fertility, previously knowing its physico-chemical characteristics to infer the proper management, be methods of correction and fertilization, or conservation practices that minimize the degradation and erosion of

\footnotetext{
${ }^{1}$ Departamento de Fitotecnia, Universidade Federal do Ceará - UFC, Fortaleza, CE, Brasil.

${ }^{2}$ Departamento de Agronomia, Instituto Federal de Educação, Ciência e Tecnologia do Ceará - IFCE, Limoeiro do Norte, CE, Brasil.

${ }^{3}$ Departamento de Fitotecnia, Universidade Federal Rural do Semiárido - UFERSA, Mossoró, RN, Brasil.

${ }^{4}$ Departamento de Agronomia, Universidade Federal de Alagoas - UFAL, Maceió, AL, Brasil.

${ }_{5}^{5}$ Departamento de Engenharia Agrícola, Universidade Federal do Ceará - UFC, Fortaleza, CE, Brasil.

Autor correspondente: Kássio Ewerton Santos Sombra, Departamento de Fitotecnia, Universidade Federal do Ceará - UFC, Avenida Mister Hull, 2977, Bloco 805, Campus do Pici, CEP 60356-001, Fortaleza, CE, Brasil. kassiosombra@gmail.com
} 
agricultural soils, which tends to aggravate in the hot and dry climate. The study evaluated and compared the physical and chemical attributes of Russas orange orchards (Citrus sinensis L. Osbeck) (Rutaceae), with productive or juvenile plants, in family farms in the semi-arid region of Ceará, Brazil. The physical and chemical characteristics evaluated consisted of particle size, particle and soil density, determining the textural class, in addition to the organic carbon and organic matter contents; macros and micronutrients, such as available phosphorus and potassium, or exchangeable sodium, calcium, magnesium and aluminum contents; as well as, essential micronutrients. By determining the sum of the bases, the cation exchange capacity, exchangeable base saturation and sodium saturation. Soils of productive and juvenile orchards did not differ significantly, with predominantly 'free' texture, with low carbon and organic matter content, as well as poor alkalinity and satisfactory macro and micronutrient levels, presenting high values for sum of bases, cation exchange capacity and base saturation, necessitating corrections and fertilization, as well as the use of conservation practices that contribute to the improvement of soil quality, and, consequently, obtaining higher yields in citrus orchards..

Index terms: citrus, fertility, Russas Orange, management.

\section{INTRODUÇÃO}

A citricultura moderna tem se desenvolvido em diversas classes de solos, mesmo em solos de baixa fertilidade natural, por exemplo, solos distróficos com baixa saturação por bases, sendo fundamental conhecer as características físico-químicas para poder inferir o manejo adequado a cada classe, permitindo a adoção de métodos de correção e adubação, além de práticas conservacionistas que minimizem a degradação e a erosão, o que pode agravar-se diante do clima quente e seco, registrado no semiárido cearense (Brady \& Weil, 2013; Dias et al., 2013; Primavesi, 2012).

O crescimento e desenvolvimento das culturas agrícolas estão diretamente ligados a fertilidade do solo, pois em conceito, é considerada como consequência da interação entre fatores físicos, químicos e biológicos do solo (Ribeiro et al., 1999; Primavesi, 2014; Primavesi, 2016). Diante da complexidade entre estas interações, e da dificuldade de conceituação, costuma-se relacionar a fertilidade aos teores de nutrientes disponíveis no solo que podem ser absorvidos pelas plantas durante o seu ciclo de vida (Malavolta, 1976; Raij, 1981, 2011; Primavesi, 2016). A análise da fertilidade é uma importante ferramenta no manejo produtivo, que permite conhecer as principais características químicas do solo, e orientar o planejamento e o manejo produtivo, através da observação dos níveis de nutrientes, potencialmente, disponíveis para as raízes das plantas (suficiência e/ou deficiência), assim como, condições adversas ao desenvolvimento vegetal, como acidez, salinidade, e orientar correções e adubações, que interferem sobre a produtividade das plantas, como de frutos cítricos (Bouaziz et al., 2011; Abbas et al., 2013; Brady \& Weil, 2013).
As plantas cítricas tendem a se desenvolver melhor em solos de textura 'franca (média)', porém, a citricultura moderna e a adoção de porta-enxertos adaptados aos principais estresses abióticos, permitem que os citros sejam cultivados comercialmente em solos de diferentes classes texturais, apesar de não tolerarem solos impermeáveis (Mattos Junior et al., 2009).

As plantas cítricas possuem uma demanda variável de carbono, oxigênio, hidrogênio, nitrogênio, fósforo, potássio, cálcio, magnésio, enxofre, zinco, cobre, boro, manganês, cloro e ferro, entre outros, retirados pelos mecanismos vegetais da água, do ar e do próprio solo, e utilizados para os processos de crescimento e desenvolvimento vegetal (Mattos Junior et al., 2009; Hondebrink et al., 2017). Assim sendo, Mattos Junior et al. (2009) buscaram estabelecer recomendações de adubações que suprissem as demandas nutricionais das plantas cítricas em seus diferentes estádios de produção, distinguindo-as quanto ao plantio (fundação), cobertura (formação - plantas no estádio juvenil) e produção (plantas adultas no estádio reprodutivo). No entanto, na adubação de pomares cítricos, Mattos Junior et al. (2009) sugerem como critério de ajuste, além dos teores de nutrientes disponíveis no solo, a produtividade esperada pelas culturas e o teor de nitrogênio nas folhas, atribuindo-se uma demanda extra de nutrientes para suprir o desenvolvimento dos frutos, além dos nutrientes essenciais ao crescimento de folhas, ramos e raízes.

Diante disto, o trabalho avaliou e comparou os atributos físicos e químicos dos solos de pomares de laranja de Russas (Citrus sinensis L. Osbeck) (Rutaceae), com plantas produtivas ou juvenis, na agricultura familiar do semiárido cearense. 


\section{MATERIAL E MÉTODOS}

O trabalho foi desenvolvido entre setembro de 2016 e março de 2017, em pomares cítricos produtivos e juvenis localizados nas zonas rurais dos municípios de Limoeiro do Norte, Quixeré e Russas, no Baixo Jaguaribe, Ceará.

A região do Baixo Jaguaribe apresenta clima classificado como seco e muito quente, do tipo BSw'h' (Köppen) (Alvares et al., 2013), inserindo-se no semiárido nordestino, e no bioma caatinga. Entre os dados climáticos destacam-se a temperatura média anual de $27,1^{\circ} \mathrm{C}$, oscilando entre $22,3{ }^{\circ} \mathrm{C}$ e $34,8^{\circ} \mathrm{C}$, associada a umidade relativa (UR) do ar variando de $44 \%$ a $85 \%$, e regime pluviométrico médio de $857,7 \mathrm{~mm}$, com variação histórica entre 548 e $992 \mathrm{~mm}$, caracterizando-se por chuvas irregulares, 'mal' distribuídas ao longo do ano, e duas estações bem definidas, uma seca entre junho e janeiro, e uma chuvosa, de fevereiro a maio (verão e outono) (INMET, 2018).

No Baixo Jaguaribe predominam os 'Neossolos Flúvicos (RY)', considerados solos minerais não hidromórficos, com alto potencial agrícola quando apresentam textura média e boa drenagem. Acima dos solos, a vegetação nativa é constituída principalmente por espécimes da palmeira nativa, símbolo do estado do Ceará, as carnaubeiras (Copernicia prunifera Mill. H. E. Moore) (Arecaceae), que formam extensos carnaubais, intercalados por áreas preservadas de caatinga e pequenas propriedades conduzidas em sua maioria sob base da agricultura familiar (Sombra et al., 2016; Sombra et al., 2018).

Adotou-se delineamento inteiramente casualizado (DIC), em esquema fatorial $2 \times 2$, com quatro tratamentos e cinco repetições, totalizando 20 amostras úteis por unidade amostral (pomar) de 0,5 hectare, aproximadamente. Os fatores consistiram da origem (pomares produtivos ou juvenis) e a profundidade de coleta, listando-se: solos de pomares produtivos com laranjeiras de Russas (pés francos) e solos de pomares juvenis com clones de laranjeiras BRS Russas (C. sinensis), enxertadas sobre diferentes porta-enxertos, em duas profundidades (0 a $20 \mathrm{~cm}$ e 20 a $40 \mathrm{~cm}$ ).

Os pomares produtivos apresentavam plantas com idade entre 10 e 30 anos, geralmente, espaçadas $7 \times 7 \mathrm{~m}$, recebendo adubações orgânicas de cobertura com estercos e restos vegetais, coroamento e cobertura morta, indução de florescimento por estresse hídrico, eliminação de brotos 'ladrões' e irrigação por superfície (Sombra et al., 2018). Já os pomares juvenis eram constituídos de combinações entre oito clones de laranja BRS Russas e diferentes porta-enxertos, por exemplo, os citrandarins
Riverside, Indio e San Diego (Citrus sunki Hayata hort. ex Tanaka $\times$ Poncirus trifoliata L. Raf.) (Rutaceae), com 3 anos de idade, espaçadas $5 \times 2 \mathrm{~m}$, adubadas com compostos orgânicos a base de esterco bovino e restos vegetais, além de irrigadas por microaspersão, com apoio da Empresa Brasileira de Pesquisa Agropecuária (Embrapa).

Analisaram-se as características físicas associadas a composição granulométrica (areia, silte e argila), densidade das partículas e do solo, determinando-se a classe textural dos solos dos pomares cítricos (Embrapa, 2011). As características químicas avaliadas foram: carbono orgânico (C.O.); matéria orgânica (M.O.); potencial hidrogeniônico (pH); fósforo $(\mathrm{P})$ e potássio $(\mathrm{K})$ disponíveis; sódio $(\mathrm{Na})$, cálcio $(\mathrm{Ca})$, magnésio $(\mathrm{Mg})$ e alumínio trocáveis $(\mathrm{Al})$; acidez potencial $(\mathrm{H}+\mathrm{Al})$; disponibilidade dos micronutrientes [cobre $(\mathrm{Cu})$, ferro $(\mathrm{Fe})$, manganês $(\mathrm{Mn})$, zinco $(\mathrm{Zn})$ e boro (B)]; soma das bases (SB); capacidade de troca de cátions à $\mathrm{pH} 7,0(\mathrm{CTC})$; porcentagem de saturação por bases trocáveis $(\mathrm{V})$; porcentagem de saturação por sódio (PST) e condutividade elétrica (CE) (Embrapa, 2009; Embrapa, 2017; Mapa, 2014).

Cada amostra útil constituiu-se de uma amostra composta, elaborada a partir de 20 sub amostras simples coletadas aleatoriamente com auxílio de um trado tipo 'tubo', adotando-se amostragem sequencial. As amostras simples (sub amostras) foram misturadas de forma homogênea, retirando-se uma amostra composta com cerca de $1,5 \mathrm{Kg}$, acondicionada em saco plástico e levado ao Laboratório de Solos, Água e Tecidos Vegetais - LABSAT do Instituto Federal de Educação, Ciência e Tecnologia do Ceará - IFCE para realização das determinações analíticas. No Laboratório, as amostras foram recepcionadas, retiradas dos sacos e distribuídas em bandejas metálicas para secagem em estufa $\left(45^{\circ} \mathrm{C}\right)$, determinando-se a fração menor que $2 \mathrm{~mm}$, denominada terra fina seca em estufa (TFSE) (Embrapa, 2011).

Inicialmente, determinou-se a composição granulométrica por meio do 'método de pipeta', utilizando-se os valores das proporções de areia, silte e argila, para realizar a classificação com auxílio do triângulo textural (Nogueira \& Souza, 2005; Embrapa, 2009). A densidade aparente (Ds) foi calculada determinando-se o peso de solo compactado necessário para completar o volume de uma proveta de $100 \mathrm{ml}$, enquanto a densidade das partículas (Dp) foi determinada com base no volume de álcool necessário para completar a capacidade de um balão volumétrico, contendo terra fina seca em estufa (TFSE) (Embrapa, 2011).

A determinação do carbono orgânico (C.O.) foi realizada através da oxidação da matéria orgânica via úmida, 
utilizando dicromato de potássio em meio sulfúrico, e empregando o calor desprendido do ácido sulfúrico como fonte de energia. Ao fim, calculou-se a percentagem de matéria orgânica (M.O.) multiplicando o resultado do carbono orgânico pelo fator 1,724 , para obter o teor de matéria orgânica do solo, expresso em $\mathrm{g} \mathrm{dm}^{-3}$ (Quaggio \& Raij, 1979).

O potencial hidrogeniônico $(\mathrm{pH})$ foi determinado de forma eletrônica por meio de eletrodo combinado imerso em suspensão solo: líquido $\left(\mathrm{H}_{2} \mathrm{O}, \mathrm{CaCl}_{2} ; 1: 2,5\right)$, utilizando potenciômetro de bancada para realizar leituras em triplicata (Embrapa, 2017).

$\mathrm{O}$ teor de fósforo $(\mathrm{P})$ disponível foi calculado mediante extração com Mehlich ( $\mathrm{HCl} 0,05 \mathrm{~N}+\mathrm{H}_{2} \mathrm{SO}_{4} 0,025 \mathrm{~N}$ ), realizando a leitura direta no fotocolorímetro, e obtendo os valores expressos em mg dm${ }^{-3}$. Os teores de sódio $\left(\mathrm{Na}^{+}\right)$ e potássio $\left(\mathrm{K}^{+}\right)$trocáveis também foram extraídos com Mehlich $^{-1}$, realizando a leitura através de espectrofotometria de chama, enquanto os teores de $\mathrm{Ca}^{2+}$ e $\mathrm{Mg}^{2+}$ do solo foram obtidos por extração com $\mathrm{KCl} 1 \mathrm{~N}$ e titulação com EDTA $0,025 \mathrm{~N}$, similar a determinação do teor de $\mathrm{Al}^{3+}$ do solo por extração com $\mathrm{KCl} 1 \mathrm{~N}$, porém, titulação com $\mathrm{NaOH} 0,025 \mathrm{~N}$, obtendo-se os resultados expressos em $\mathrm{cmol}_{\mathrm{c}} / \mathrm{dm}^{3}$ (Embrapa, 2009; Embrapa, 2011).

Os teores de micronutrientes disponíveis $\left(\mathrm{Cu}^{+}, \mathrm{Fe}^{2+}, \mathrm{Mn}^{2+}, \mathrm{Zn}^{2+}\right.$ e B) foram determinados por extração com Mehlich $\left(\mathrm{HCl} 0,05 \mathrm{~N}+\mathrm{H}_{2} \mathrm{SO}_{4} 0,025 \mathrm{~N}\right)$, obtendo resultados em $\mathrm{mg} \mathrm{dm}^{-3}$ (Embrapa, 2011).

A acidez potencial $\left(\mathrm{H}+\mathrm{Al}^{3+}\right)$ foi extraída utilizando acetato de cálcio $0,5 \mathrm{~mol} \mathrm{~L}^{-1}$, à $\mathrm{pH} 7,0$, realizando a determinação por titulação com $\mathrm{NaOH} 0,025 \mathrm{~mol} \mathrm{~L}^{-1}$, obtendo valores em $\mathrm{cmolc} \mathrm{dm}^{-3}$. A soma de bases (S.B.) foi determinada utilizando a fórmula a seguir, e obtendo resultados em cmolc dm ${ }^{-3}$ (Embrapa, 2011):

S.B. $=\mathrm{Ca}^{2+}+\mathrm{Mg}^{2+}+\mathrm{K}^{+}+\mathrm{Na}^{+}$

(Equação 1)

Calculou-se a capacidade de troca catiônica à $\mathrm{pH}$ 7,0 (CTC), aplicando-se a seguinte fórmula, e obtendo valores em cmolc dm ${ }^{-3}$ (Embrapa, 2011):

$C T C=S . B .+\left(H^{+}+A l^{3+}\right)$

(Equação 2)

A porcentagem de saturação por bases trocáveis (V) foi determinada através da aplicação da fórmula (Embrapa, 2011):

$V=100 \times S . B . / C T C$

(Equação 3)

A porcentagem de sódio trocável (PST) foi obtida pela aplicação da fórmula proposta por Richards (1954): $P S T=100 \times\left(\mathrm{Na}^{+}\right) / \mathrm{CTC}$

(Equação 4)
E por último, mensurou-se a condutividade elétrica (CE) do solo através de leitura direta, $\mathrm{em} \mathrm{mS} \mathrm{cm}^{-1}$, utilizando condutivímetro de bancada (Embrapa, 2017).

Os dados obtidos, para todas as variáveis, foram submetidos à análise de variância (ANOVA), comparando-se as médias pelo teste $t$ (LSD) ao nível de $5 \%$ de probabilidade $(\mathrm{p} \leq 0,05)$, utilizando software estatístico SISVAR ${ }^{\circledR}$ (Ferreira, 2011).

\section{RESULTADOS E DISCUSSÃO}

Constataram-se teores médios de argila na maioria das amostras, classificando-se $75 \%$ dos solos dos pomares produtivos na classe textural 'franca', e 50\% dos solos dos pomares juvenis, porém, constatando-se também solos de textura 'franco siltosa' entre os solos dos pomares produtivos, e 'franco argilo siltosa' entre os juvenis ( $\mathrm{SiBS}$ ) (Tabela 1).

As principais culturas agrícolas modernas são cultivadas, preferencialmente, em solos de textura 'franca', devido a sua facilidade de manejo, por apresentar balanço satisfatório entre os teores de areia, silte e argila, responsáveis por proporcionar uma drenagem da água eficiente para as principais culturas comerciais (Embrapa, 2013).

Os valores obtidos para densidade do solo, definida como a relação entre a massa de uma amostra de solo seca a $105^{\circ} \mathrm{C}$ e a soma dos volumes ocupados pelas partículas e pelos poros nos pomares produtivos, ou juvenis, mantiveram-se entre 1,25 a $1,60 \mathrm{~g} \mathrm{~cm}^{-3}$, compreendidos como solos arenosos (Corrêa et al., 2010; Brady \& Weil, 2013; Gubiani et al., 2015). Os solos com características similares tendem a ter textura levemente suave, parcialmente arenosa e pegajosa., desintegrando-se facilmente quando compactado, o que os tornam fáceis de manejar para cultivo de citros (Brady \& Weil, 2013; Primavesi, 2016).

Os cultivos de plantas cítricas em solos de textura 'franca', geralmente, são favorecidos pela boa drenagem que estes solos proporcionam, permitindo que a água desça (verticalmente no solo) em uma velocidade suficiente para evitar o excesso de umidade na rizosfera, e possibilitar a absorção pelos sistemas radiculares das plantas cítricas, quando comparados a solos de menor permeabilidade, minimizando, de forma indireta, riscos de podridões radiculares ou proliferações de fitopatógenos de solo (Minatel et al., 2006; Corrêa et al., 2010).

Apesar da enorme importância da textura do solo no desenvolvimento das plantas cítricas, como é possível constatar no desempenho de alguns citros em solos de 
Tabela 1. Composição granulométrica dos solos dos pomares cítricos produtivos e juvenis, Limoeiro do Norte/ $\mathrm{CE}-2018$

\begin{tabular}{|c|c|c|c|c|c|c|}
\hline \multirow{3}{*}{$\begin{array}{c}\text { Tratamentos } \\
\text { (pomares cítricos) }\end{array}$} & \multirow{2}{*}{ Areia grossa } & \multirow{2}{*}{ Areia fina } & \multirow{2}{*}{ Silte } & \multirow{2}{*}{ Argila } & \multicolumn{2}{|c|}{ Densidade } \\
\hline & & & & & Solo & Partícula \\
\hline & \multicolumn{4}{|c|}{----------------------- g kg$^{-1}$--------------------- } & \multicolumn{2}{|c|}{------ $\mathrm{g} \mathrm{cm}^{-3}$} \\
\hline Produtivos $0-20 \mathrm{~cm}$ & $170,5 \mathrm{a}$ & $424,0 \mathrm{a}$ & $247,5 \mathrm{a}$ & $158,0 \mathrm{a}$ & $1,37 \mathrm{a}$ & $2,47 \mathrm{a}$ \\
\hline Produtivos $20-40 \mathrm{~cm}$ & $148,0 \mathrm{a}$ & $372,7 \mathrm{a}$ & $286,5 \mathrm{a}$ & 193,0 a & $1,38 \mathrm{a}$ & $2,52 \mathrm{a}$ \\
\hline Juvenis $0-20 \mathrm{~cm}$ & $111,2 \mathrm{a}$ & $314,7 \mathrm{a}$ & 345,7 a & $228,2 \mathrm{a}$ & $1,36 \mathrm{a}$ & $2,52 \mathrm{a}$ \\
\hline Juvenis $20-40 \mathrm{~cm}$ & $126,2 \mathrm{a}$ & $344,2 \mathrm{a}$ & $317,7 \mathrm{a}$ & $211,7 \mathrm{a}$ & $1,32 \mathrm{a}$ & $2,44 \mathrm{a}$ \\
\hline Média geral & 139,00 & 363,93 & 299,37 & 197,75 & 1,18 & 2,48 \\
\hline C.V. $(\%)$ & 97,28 & 51,93 & 41,33 & 51,77 & 8,86 & 2,88 \\
\hline Valor de F & $0,077^{\mathrm{ns}}$ & $0,183^{\mathrm{ns}}$ & $0,293^{\text {ns }}$ & $0,253^{\text {ns }}$ & $0,333^{\text {ns }}$ & $1,24^{\mathrm{ns}}$ \\
\hline Valor-p & 0,7862 & 0,6767 & 0,5981 & 0,6240 & 0,5740 & 0,3380 \\
\hline
\end{tabular}

As médias seguidas pela mesma letra não diferem estatisticamente entre si. NS= Não significativo.

textura franca, a adoção de porta-enxertos adaptados ou tolerantes às diferentes condições edáficas, e o planejamento de sistemas de irrigação localizada, tem contribuído para superar os desafios, e permitir que a citricultura seja praticada em todas as regiões do país (Mattos Junior et al., 2009).

A literatura específica para interpretação e recomendações para o cultivo de citros em condições semiáridas ainda é escassa, diante disto, buscou-se interpretar os resultados com base no proposto pela Comissão de Fertilidade do Solo do Estado de Minas Gerais - $5^{\text {a }}$ Aproximação, conforme Tabelas 2 a 5 (Ribeiro et al., 1999).

Os pomares cítricos produtivos e juvenis apresentaram baixos teores para o carbono (C.O.) e matéria orgânica (M.O.), alcalinidade fraca $(\mathrm{pH})$, e teores satisfatórios de fósforo disponível $(\mathrm{P})$, potássio $\left(\mathrm{K}^{+}\right)$, cálcio $\left(\mathrm{Ca}^{2+}\right) \mathrm{e}$ magnésio trocável $\left(\mathrm{Mg}^{2+}\right)$, assim como, soma das bases (SB), capacidade de troca catiônica (CTC) e saturação por bases (V) (Tabelas 6 e 7).

As variações observadas, e que consequentemente, elevaram o coeficiente de variação (C.V.), possivelmente, estão associadas as variações no manejo cultural das propriedades cítricas, constatando-se que os baixos índices de carbono (C) e matéria orgânica (M.O.), podem dever-se, em parte, ao intervalo de tempo entre realizações de adubações orgânicas, que variaram entre 6 e 12 meses, além do uso de cobertura morta em superfície, que apesar de funcionarem como técnicas condicionadoras do solo, mantendo-o coberto e protegendo-o por um determinado período, não mantinham-se até a nova adubação, ou adição de cobertura (palhada), expondo as camadas superficiais do solo aos intempéries típicos do semiárido, possivelmente, reduzindo os teores de $\mathrm{C}$ e $\mathrm{MO}$, assim como, a atividade biológica, principalmente em períodos de maior radiação solar e temperatura (Primavesi, 2012; Alves et al., 2016; Santos et al., 2016; Fernandes et al., 2016; Primavesi, 2016).

A disponibilidade adequada de cálcio $(\mathrm{Ca})$ pode melhorar as condições de crescimento radicular, estimular a atividade microbiana, auxiliar na disponibilidade do molibdênio (Mo) e na absorção de outros nutrientes, como magnésio $(\mathrm{Mg})$, que atua como componente da molécula de clorofila, além de influenciar no movimento de distribuição dos carboidratos das folhas para outras partes da planta, e estimular a captação e transporte do fósforo $(\mathrm{P})$ na planta. Ao considerar as interações entre os nutrientes no solo, pode inferir que o seu balanço interfere diretamente sobre a desenvolvimento e o rendimento das plantas cítricas, induzindo a planta capacidade de tolerar estresses bióticos e abióticos, como por exemplo, competição com outras plantas, ataque de artrópodes-praga ou fitopatógenos (Dechen \& Nachtigall, 2006; Abbas et al., 2013; Brady \& Weil, 2013).

O equilíbrio observado entre os macronutrientes, também pôde ser observado entre os teores de micronutrientes nos pomares de laranja de Russas (C. sinensis), registrando-se valores similares de cobre $(\mathrm{Cu})$, zinco $(\mathrm{Zn})$, manganês $(\mathrm{Mn})$, ferro (Fe) e Boro (B), oscilando entre bons e muito bons, não se registrando diferença significativa entre os produtivos e juvenis, ou profundidades (Tabela 8).

A disponibilidade de micronutrientes, como manganês (Mn) e zinco (Zn) (Hippler et al., 2014), ou por exemplo cobre $(\mathrm{Cu})$, essencial para a fotossíntese e respiração mitocondrial, assim como, para o metabolismo do nitrogênio 
Tabela 2. Classes de interpretação para o potencial hidrogeniônico $(\mathrm{pH})^{1}$

\begin{tabular}{|c|c|c|c|c|c|c|}
\hline \multicolumn{7}{|c|}{ Classificação química } \\
\hline $\begin{array}{l}\text { Acidez muito } \\
\text { elevada }\end{array}$ & $\begin{array}{l}\text { Acidez } \\
\text { elevada }\end{array}$ & Acidez média & Acidez fraca & Neutra & $\begin{array}{l}\text { Alcalinidade } \\
\text { fraca }\end{array}$ & $\begin{array}{c}\text { Alcalinidade } \\
\text { elevada }\end{array}$ \\
\hline$<4,5$ & $4,5-5,0$ & $5,1-6,0$ & $6,1-6,9$ & 7,0 & $7,1-7,8$ & $>7,8$ \\
\hline \multicolumn{7}{|c|}{ Classificação agronômica ${ }^{2}$} \\
\hline Muito baixo & & Baixo & Bom & Alto & \multicolumn{2}{|c|}{ Muito alto } \\
\hline$<4,5$ & & $4,5-5,4$ & $5,5-6,0$ & $6,1-7,0$ & & 7,0 \\
\hline
\end{tabular}

Fonte: Adaptado de Ribeiro et al. (1999). ${ }^{1} \mathrm{pH}\left(\mathrm{H}_{2} \mathrm{O}, \mathrm{CaCl}_{2} ; 1: 2,5\right) .{ }^{2} \mathrm{~A}$ qualificação utilizada indica adequado (bom) ou inadequado (muito baixo e baixo, alto e muito alto).

Tabela 3. Classes de interpretação de fertilidade do solo para a matéria orgânica e para o complexo de troca catiônica

\begin{tabular}{|c|c|c|c|c|c|c|}
\hline \multirow{2}{*}{ Característica } & \multicolumn{6}{|c|}{ Classificação } \\
\hline & Unidade $^{1}$ & Muito baixo & Baixo & Médio $^{2}$ & Bom & Muito bom \\
\hline Carbono orgânico $^{3}$ & $\mathrm{~g} / \mathrm{kg}$ & $\leq 4$ & $4,1-11,6$ & $11,7-23,2$ & $23,3-40,6$ & $>40,6$ \\
\hline Matéria orgânica $^{3}$ & $\mathrm{~g} / \mathrm{kg}$ & $\leq 7$ & $7,1-20$ & $20,1-40$ & $40,1-70$ & $>70$ \\
\hline Cálcio trocável ${ }^{4}$ & $\mathrm{mmol} / \mathrm{dm}^{3}$ & $\leq 4$ & $4,1-12$ & $12,1-24$ & $24,1-40$ & $>40$ \\
\hline Magnésio trocável ${ }^{4}$ & $\mathrm{mmol} / \mathrm{dm}^{3}$ & $\leq 1,5$ & $1,6-4,5$ & $4,6-9$ & $9,1-15$ & $>15$ \\
\hline Acidez trocável ${ }^{4}$ & $\mathrm{mmol}_{\mathrm{c}}^{\mathrm{c}} / \mathrm{dm}^{3}$ & $\leq 2$ & $2,1-5$ & $5,1-10$ & $10,1-20^{11}$ & $>2^{11}$ \\
\hline Soma de bases ${ }^{5}$ & $\mathrm{mmol}_{\mathrm{c}}^{\mathrm{c}} / \mathrm{dm}^{3}$ & $\leq 6$ & $6,1-18$ & $18,1-36$ & $36,1-60$ & $>60$ \\
\hline Acidez potencial $^{6}$ & $\mathrm{mmol}_{\mathrm{c}}^{\mathrm{c}} / \mathrm{dm}^{3}$ & $\leq 10$ & $10,1-25$ & $25,1-50$ & $50,1-90^{11}$ & $>90^{11}$ \\
\hline CTC efetiva ${ }^{7}$ & $\mathrm{mmol}_{\mathrm{c}}^{\mathrm{c}} / \mathrm{dm}^{3}$ & $\leq 8$ & $8,1-23$ & $23,1-46$ & $46,1-80$ & $>80$ \\
\hline $\mathrm{CTC} \mathrm{pH} 7^{8}$ & $\mathrm{mmol}_{\mathrm{c}}^{\mathrm{c}} / \mathrm{dm}^{3}$ & $\leq 16$ & $16,1-43$ & $43,1-86$ & $86,1-150$ & $>150$ \\
\hline Saturação por $\mathrm{Al}^{3+9}$ & $\%$ & $\leq 15$ & $15,1-30$ & $30,1-50$ & $50,1-75^{11 /}$ & $>75^{11}$ \\
\hline Saturação por bases ${ }^{10}$ & $\%$ & $\leq 20$ & $20,1-40$ & $40,1-60$ & $60,1-80$ & $>80$ \\
\hline
\end{tabular}

Fonte: Adaptado de Ribeiro et al. (1999). ${ }^{1} \mathrm{dag} / \mathrm{kg} \times 10=\mathrm{g} / \mathrm{kg} ; \mathrm{cmol}_{\mathrm{c}} / \mathrm{dm}^{3} \times 10=\mathrm{mmol} / \mathrm{dm}^{3} .{ }^{2} \mathrm{O}$ limite superior desta classe indica o nível crítico. ${ }^{3}$ Método Walkley \& Black; M.O. $=1,724 \times$ C.O. ${ }^{4}$ Método $\mathrm{KCl} 1 \mathrm{~mol} / \mathrm{L} .{ }^{5} \mathrm{SB}=\mathrm{Ca}^{2+}+\mathrm{Mg}^{2+}+\mathrm{K}^{+}+\mathrm{Na}^{+}$. ${ }^{6} \mathrm{H}+\mathrm{Al}$, Método $\mathrm{Ca}(\mathrm{OAc})_{2} 0,5 \mathrm{~mol} / \mathrm{L}, \mathrm{pH} 7 .{ }^{7} \mathrm{t}=\mathrm{SB}+\mathrm{Al}^{3+} \cdot{ }^{8} \mathrm{~T}=\mathrm{SB}+(\mathrm{H}+\mathrm{Al}) \cdot{ }^{9} \mathrm{~m}=100 \mathrm{Al}^{3+} / \mathrm{t} .{ }^{10} \mathrm{~V}=100 \mathrm{SB} / \mathrm{T} .{ }^{11} \mathrm{~A}$ interpretação destas características, nestas classes, deve ser alta e muito alta em lugar de bom e muito bom.

Tabela 4. Classes de interpretação da disponibilidade para o fósforo $(\mathrm{P})$ de acordo com o teor de argila do solo e para o potássio $(\mathrm{K})$

\begin{tabular}{|c|c|c|c|c|c|}
\hline \multirow{3}{*}{ Característica } & \multicolumn{5}{|c|}{ Classificação } \\
\hline & Muito baixo & Baixo & Médio $^{2}$ & Bom & Muito bom \\
\hline & \multicolumn{5}{|c|}{------------------------------------ $\left(\mathrm{mg} / \mathrm{dm}^{3}\right)^{1}$------------------------------------ } \\
\hline Argila (\%) & \multicolumn{5}{|c|}{ Fósforo disponível $(\mathrm{P})^{2}$} \\
\hline $60-100$ & $<2,7$ & $2,8-5,4$ & $5,5-8,0$ & $8,1-12,0$ & $>12,0$ \\
\hline $35-60$ & $<4,1$ & $4,1-8,0$ & $8,1-12,0$ & $12,1-18,0$ & $>18,0$ \\
\hline $15-35$ & $<6,7$ & $6,7-12,0$ & $12,1-20,0$ & $20,1-30,0$ & $>30,0$ \\
\hline \multirow[t]{3}{*}{$0-15$} & $<10,1$ & $10,1-20,0$ & $20,1-30,0$ & $30,1-45,0$ & $>45,0$ \\
\hline & \multicolumn{5}{|c|}{ Potássio disponível $(\mathrm{K})^{2}$} \\
\hline & $<16$ & $16-40$ & $41-70$ & $71-120$ & $>120$ \\
\hline
\end{tabular}

Fonte: Adaptado de Ribeiro et al. (1999). ${ }^{1} \mathrm{mg} / \mathrm{dm}^{3}=$ p.p.m. (m/v). ${ }^{2}$ Método Mehlich. 
Tabela 5. Classes de interpretação da disponibilidade para os micronutrientes

\begin{tabular}{|c|c|c|c|c|c|}
\hline \multirow{3}{*}{ Característica } & \multicolumn{5}{|c|}{ Classificação } \\
\hline & Muito baixo & Baixo & Médio $^{1}$ & Bom & Muito bom \\
\hline & \multicolumn{5}{|c|}{--------------------------------- $\left(\mathrm{mg} / \mathrm{dm}^{3}\right)^{2}$-------------------------------- } \\
\hline Cobre $(\mathrm{Cu})^{3}$ & $<0,4$ & $0,4-0,7$ & $0,8-1,2$ & $1,3-1,8$ & $>1,8$ \\
\hline Zinco $(\mathrm{Zn})^{3}$ & $<0,5$ & $0,5-0,9$ & $1,0-1,5$ & $1,6-2,2$ & $>2,2$ \\
\hline Manganês $(\mathrm{Mn})^{3}$ & $<3$ & $3-5$ & $6-8$ & $9-12$ & $>12$ \\
\hline Ferro $(\mathrm{Fe})^{3}$ & $<9$ & $9-18$ & $19-30$ & $31-45$ & $>45$ \\
\hline Boro $(B)^{4}$ & $<0,16$ & $0,16-0,35$ & $0,36-0,60$ & $0,61-0,90$ & $>0,90$ \\
\hline
\end{tabular}

Fonte: Adaptado de Ribeiro et al. (1999). ${ }^{1} \mathrm{O}$ limite superior desta classe indica o nível crítico. ${ }^{2} \mathrm{mg} / \mathrm{dm}^{3}=$ p.p.m. (m/v). ${ }^{3}$ Método Mehlich. ${ }^{4}$ Método água quente.

Tabela 6. Valores de carbono orgânico (C.O.), matéria orgânica (M.O.), potencial hidrogeniônico (pH), fósforo disponível $(\mathrm{P})$, potássio trocável $\left(\mathrm{K}^{+}\right)$, cálcio trocável $\left(\mathrm{Ca}^{2+}\right)$, magnésio trocável $\left(\mathrm{Mg}^{2+}\right)$ e sódio trocável $\left(\mathrm{Na}^{+}\right)$, dos solos dos pomares cítricos, Limoeiro do Norte/CE - 2018

\begin{tabular}{|c|c|c|c|c|c|c|c|c|}
\hline \multirow{2}{*}{ Tratamentos (pomares cítricos) } & C.O. & M.O. & \multirow{2}{*}{$\mathrm{pH}$} & \multirow{2}{*}{$\frac{\mathrm{P}}{\mathrm{mg} / \mathrm{dm}^{3}}$} & $\mathrm{~K}$ & $\mathrm{Ca}$ & $\mathrm{Mg}$ & $\mathrm{Na}$ \\
\hline & \multicolumn{2}{|c|}{----- g/kg ----- } & & & \multicolumn{4}{|c|}{----------- mmolc/dm³ ------------- } \\
\hline Produtivos $0-20 \mathrm{~cm}$ & $11,1 \mathrm{a}$ & $19,1 \mathrm{a}$ & $7,52 \mathrm{a}$ & $74,25 \mathrm{a}$ & $6,64 a$ & $67,42 \mathrm{a}$ & $27,75 \mathrm{a}$ & $12,07 \mathrm{a}$ \\
\hline Produtivos $20-40 \mathrm{~cm}$ & $6,3 a$ & $11,0 \mathrm{a}$ & $7,25 \mathrm{a}$ & $58,75 \mathrm{a}$ & $4,19 a$ & $74,12 \mathrm{a}$ & $50,47 \mathrm{a}$ & $18,49 \mathrm{a}$ \\
\hline Juvenis $0-20 \mathrm{~cm}$ & $12,2 \mathrm{a}$ & $21,1 \mathrm{a}$ & $7,32 \mathrm{a}$ & $78,75 \mathrm{a}$ & $7,60 \mathrm{a}$ & $73,40 \mathrm{a}$ & $50,12 \mathrm{a}$ & $19,44 a$ \\
\hline Juvenis $20-40 \mathrm{~cm}$ & $10,3 \mathrm{a}$ & $17,8 \mathrm{a}$ & $7,32 \mathrm{a}$ & $56,25 \mathrm{a}$ & $4,00 \mathrm{a}$ & $82,30 a$ & $51,52 \mathrm{a}$ & $20,86 a$ \\
\hline Média geral & 9,93 & 17,25 & 7,37 & 67,00 & 5,56 & 74,37 & 45,18 & 17,75 \\
\hline C.V. $(\%)$ & 42,92 & 42,77 & 12,06 & 72,49 & 58,43 & 31,63 & 48,90 & 66,74 \\
\hline Valor de F & $0,58^{\text {ns }}$ & $0,46^{\mathrm{ns}}$ & $0,31^{\mathrm{ns}}$ & $0,02^{\mathrm{ns}}$ & $0,05^{\mathrm{ns}}$ & $0,27^{\mathrm{ns}}$ & $1,01^{\mathrm{ns}}$ & $0,17^{\mathrm{ns}}$ \\
\hline Valor-p & 0,460 & 0,510 & 0,584 & 0,887 & 0,821 & 0,845 & 0,333 & 0,680 \\
\hline
\end{tabular}

As médias seguidas pela mesma letra não diferem estatisticamente entre si. NS= Não significativo.

Tabela 7. Valores de acidez trocável $\left(\mathrm{Al}^{3+}\right)$, acidez potencial $(\mathrm{H}+\mathrm{Al})$, soma das bases $(\mathrm{SB}), \mathrm{CTC}$ à $\mathrm{pH} 7,0$, saturação por bases $(\mathrm{V})$, porcentagem de sódio trocável (PST), saturação por $\mathrm{Al}^{3+}(\mathrm{m})$ e condutividade elétrica $(\mathrm{CE})$ dos solos dos pomares cítricos, Limoeiro do Norte/CE - 2018

\begin{tabular}{|c|c|c|c|c|c|c|c|c|}
\hline \multirow{2}{*}{$\begin{array}{c}\text { Tratamentos } \\
\text { (pomares cítricos) }\end{array}$} & $\mathrm{Al}$ & $\mathrm{H}+\mathrm{Al}$ & SB & CTC & $\mathrm{V}$ & PST & $\mathrm{m}$ & \multirow{2}{*}{$\begin{array}{c}\mathrm{CE} \\
\mathrm{dS} / \mathrm{m}\end{array}$} \\
\hline & \multicolumn{4}{|c|}{------------ mmolc/dm³ ------------- } & \multicolumn{3}{|c|}{--------- \% ----------- } & \\
\hline Produtivos $0-20 \mathrm{~cm}$ & 0 & $10,12 \mathrm{a}$ & $113,87 \mathrm{a}$ & $124,00 \mathrm{a}$ & $90,75 a$ & $9,2 \mathrm{a}$ & 0 & $1,34 \mathrm{a}$ \\
\hline Produtivos $20-40 \mathrm{~cm}$ & 0 & $23,10 \mathrm{a}$ & $147,30 \mathrm{a}$ & $170,40 \mathrm{a}$ & $88,00 \mathrm{a}$ & $10,0 \mathrm{a}$ & 0 & $1,36 \mathrm{a}$ \\
\hline Juvenis $0-20 \mathrm{~cm}$ & 0 & $17,97 \mathrm{a}$ & $151,57 \mathrm{a}$ & $169,55 \mathrm{a}$ & $90,00 \mathrm{a}$ & $11,2 \mathrm{a}$ & 0 & $1,27 \mathrm{a}$ \\
\hline Juvenis $20-40 \mathrm{~cm}$ & 0 & $17,55 \mathrm{a}$ & $158,67 \mathrm{a}$ & $176,22 \mathrm{a}$ & $90,50 \mathrm{a}$ & $12,0 \mathrm{a}$ & 0 & $1,51 \mathrm{a}$ \\
\hline Média geral & - & 17,31 & 143,00 & 160,18 & 90,31 & 10,62 & - & 1,31 \\
\hline C.V. $(\%)$ & - & 96,75 & 36,60 & 37,63 & 8,76 & 42,57 & - & 50,40 \\
\hline Valor de F & - & $0,62^{\mathrm{ns}}$ & $0,24^{\mathrm{ns}}$ & $0,43^{\mathrm{ns}}$ & $0,44^{\mathrm{ns}}$ & $0,00^{\mathrm{ns}}$ & - & $0,14^{\text {ns }}$ \\
\hline Valor-p & - & 0,443 & 0,628 & 0,522 & 0,519 & 0,997 & - & 0,712 \\
\hline
\end{tabular}

As médias seguidas pela mesma letra não diferem estatisticamente entre si. NS= Não significativo. 
Tabela 8. Teores dos micronutrientes, cobre ( $\mathrm{Cu}$ ), zinco $(\mathrm{Zn})$, manganês ( $\mathrm{Mn})$, ferro (Fe) e boro (B), nos solos dos pomares cítricos, Limoeiro do Norte/CE - 2018

\begin{tabular}{lccccc}
\hline \multirow{2}{*}{$\begin{array}{c}\text { Tratamentos } \\
\text { (pomares cítricos) }\end{array}$} & $\mathrm{Cu}$ & $\mathrm{Zn}$ & $\mathrm{Mn}$ & $\mathrm{Fe}$ & $\mathrm{B}$ \\
\cline { 2 - 6 } Produtivos 0 - $20 \mathrm{~cm}$ & $1,67 \mathrm{a}$ & $1,85 \mathrm{a}$ & $17,25 \mathrm{a}$ & $38,25 \mathrm{a}$ & $1,04 \mathrm{a}$ \\
Produtivos $20-40 \mathrm{~cm}$ & $2,10 \mathrm{a}$ & $1,77 \mathrm{a}$ & $17,37 \mathrm{a}$ & $67,50 \mathrm{a}$ & $0,78 \mathrm{a}$ \\
Juvenis 0 - $20 \mathrm{~cm}$ & $1,72 \mathrm{a}$ & $1,95 \mathrm{a}$ & $17,80 \mathrm{a}$ & $39,25 \mathrm{a}$ & $1,16 \mathrm{a}$ \\
Juvenis $20-40 \mathrm{~cm}$ & $1,55 \mathrm{a}$ & $1,47 \mathrm{a}$ & $18,20 \mathrm{a}$ & $39,25 \mathrm{a}$ & $0,99 \mathrm{a}$ \\
Média geral & 1,75 & 1,81 & 17,75 & 46,06 & 1,06 \\
C.V. (\%) & 59,48 & 60,12 & 12,65 & 61,33 & 23,53 \\
Valor de F & $0,923^{\text {ns }}$ & $0,053^{\text {ns }}$ & $0,050^{\text {ns }}$ & $0,389^{\text {ns }}$ & $1,00^{\text {ns }}$ \\
Valor-p & 0,3556 & 0,8224 & 0,8275 & 0,5447 & 0,384 \\
\hline
\end{tabular}

As médias seguidas pela mesma letra não diferem estatisticamente entre si. NS= Não significativo.

$(\mathrm{N})$, necessário à síntese de parede celular (Hansch \& Mendel, 2009), ou boro (B), que destaca-se por atuar sobre o crescimento radicular, e consequentemente, sobre o volume de solo explorado pelas raízes, maximizando o aproveitamento de água e fertilizantes (Bologna \& Vitti, 2006), pode limitar a produção dos citros nas condições tropicais, geralmente, levando a necessidade de aplicações foliares, ou via solo, de produtos compostos por estes micronutrientes (Boaretto et al., 2003; Hippler et al., 2014).

Porém a disponibilidade de $\mathrm{B}$, assim como ocorre com outros macro ou micronutrientes, é influenciada por fatores abióticos e bióticos, como a quantidade de matéria orgânica (MO) no solo, ou pelo estádio de desenvolvimento da planta cítrica, sendo que plantas em estado inicial de crescimento absorvem $\mathrm{B}$ com maior intensidade do que as plantas adultas, sendo, geralmente, baixa a mobilidade da redistribuição dos tecidos velhos para os jovens (Fernandes, 2006; Mattos Junior et al., 2009; Silva, 2016).

Os solos dos pomares apresentaram balanço satisfatório entre os macros e micronutrientes (Raij, 1981), que apesar dos baixos teores de matéria orgânica (MO), podem ser manejados para atender a demanda das plantas cítricas (Mattos Junior et al., 2003; Mattos Junior et al., 2009), destacando-se entre as práticas que podem contribuir para melhoria dos atributos dos solos, a realização de adubações orgânicas, associadas ao uso de cobertura morta, e que se realizadas de forma contínua nos pomares cítricos, sejam produtivos ou juvenis, podem elevar os teores de matéria orgânica, e consequentemente, as reações bioquímicas de decomposição e mineralização, elevando a disponibilidade de nutrientes às plantas cítricas (Mattos Junior et al., 2009; Primavesi, 2014).

\section{CONCLUSÕES}

Os solos dos pomares produtivos e juvenis não apresentaram diferença significativa, sendo predominantemente, solos de textura 'franca', com baixos teores de carbono e matéria orgânica (MO), além de alcalinidade fraca $(\mathrm{pH})$, e teores satisfatórios dos macro e micronutrientes essenciais, apresentando valores elevados para soma das bases (SB), capacidade de troca catiônica (CTC) e saturação por bases (V).

A análise dos atributos físicos e químicos do solo permitem conhecer e orientar a adoção de práticas conservacionistas que elevem os teores de $\mathrm{MO}$, como adubações orgânicas, plantio direto, cobertura morta e/ou adubação verde, e que contribuam para nutrição satisfatória da planta cítrica, elevando as produtividades dos pomares, principalmente, diante da importância da citricultura para a agricultura familiar do semiárido cearense.

\section{AGRADECIMENTOS}

Ao Conselho Nacional de Desenvolvimento Científico e Tecnológico (CNPq) pela concessão de bolsas, à Embrapa Mandioca e Fruticultura Tropical, à Embrapa Semiárido e à Secretaria de Agricultura de Russas (SEAGRI).

\section{REFERÊNCIAS}

Abbas, A., Khan, S., Hussain, N., Hanjra, M. A., \& Akbar, S. (2013). Characterizing soil salinity in irrigated 
agriculture using a remote sensing approach. Physics and Chemistry of the Earth, 55(1), 43-52.

Alvares, C. A., Stape, J. L., Sentelhas, P. C., Moraes, G., Leonardo, J., \& Sparovek, G. (2013). Köppen's climate classification map for Brazil. Meteorologische Zeitschrift, 22(6), 711-728.

Alves, H. S., Sousa, A. D. M., Mendes, E. B., Vasconcelos, I. P., \& Vieira, B. N. P. (2016). Produção orgânica de frutas e hortaliças em propriedade rural no município de Santarém-PA. Cadernos de Agroecologia, 10(3), 1-6.

Boaretto, A. E., Muraoka, T., \& Boaretto, R. M. (2003). Absorção e translocação de Mn, Zn e B aplicados via foliar em Citros. Laranja, 24(1), 177-197.

Bologna, I. R., \& Vitti, G. C. (2006). Produção e qualidade de frutos de laranjeira Pêra em função de fontes e doses de boro. Revista Brasileira de Fruticultura, 28(2), 328-330.

Bouaziz, M., Matschullat, J., \& Gloaguen, R. (2011). Improved remote sensing detection of soil salinity from a semi-arid climate in Northeast Brazil. Comptes Rendus Geoscience, 343(11), 795-803.

Brady, N., \& Weil, R. (2013). Elementos da natureza e propriedades dos solos (716 pp.). 3. ed. Porto Alegre: Editora Bookman.

Corrêa R. M., Freire M. B. S., Ferreira R. L., Silva J. A., Pessoa L. G., Miranda M. A. \& Melo D. V. (2010). Atributos físicos de solos sob diferentes usos com irrigação no semiárido de Pernambuco. Agriambi: Revista Brasileira de Engenharia Agrícola e Ambiental, 14(4): 358-365.

Dechen, A. R., \& Nachtigall, G. R. (2006). Elementos essenciais e benéficos às plantas superiores. In: M. S. Fernandes. Nutrição mineral de plantas (pp. 432-432). Viçosa: Sociedade Brasileira de Ciência do Solo.

Dias, N. S., Brígido, A. R., \& Souza, A. C. M. (2013). Manejo e conservação dos solos e da água (292 pp.). São Paulo: Editora Livraria da Física.

Empresa Brasileira de Pesquisa Agropecuária-EMBRAPA. (2009). Manual de análises químicas de solos, plantas e fertilizantes (2. ed. rev. ampl., 627 pp.). Brasília: Embrapa Informação Tecnológica.

Empresa Brasileira de Pesquisa Agropecuária-EMBRAPA. (2011). Manual de métodos de análise de solo (2. ed., 230 pp.). Rio de Janeiro: Embrapa Solos.
Empresa Brasileira de Pesquisa Agropecuária - EMBRAPA. (2013). Sistema brasileiro de classificação de solos (3 ed rev. il., 353 pp.). Rio de Janeiro: Embrapa Solos.

Empresa Brasileira de Pesquisa Agropecuária-EMBRAPA. (2017). Manual de métodos de análise de solo (3. ed. rev. atual., 573 pp.). Brasília: Embrapa.

Fernandes, C. S., Ferreira, L. L., Silva, H. E. R., Martins, A. F., \& Porto, V. C. N. (2016). A influência da cobertura morta no desempenho agronômico do coentro adubado com esterco bovino. Cadernos de Agroecologia, 10(3), 1-6.

Fernandes, M. S. (2006). Nutrição mineral de plantas (432 pp.). Viçosa: Sociedade Brasileira de Ciência do Solo.

Ferreira, D. F. (2011). Sisvar: a computer statistical analysis system. Ciência e Agrotecnologia, 5(6), 1039-1042.

Gubiani, P. I., Van Lier, Q. J., Drescher, M. S., Mezzomo, H. C., \& Veiga, M. C. C. (2015). Relação entre densidade do solo e conteúdo de água em repetidos ciclos de contração e expansão em um Latossolo. Revista Brasileira de Ciência do Solo, 39(1), 100-108.

Hansch, R., \& Mendel, R. R. (2009). Physiological functions of mineral micronutrients $(\mathrm{Cu}, \mathrm{Zn}, \mathrm{Mn}, \mathrm{Fe}, \mathrm{Ni}, \mathrm{Mo}, \mathrm{B}$, Cl). Current Opinion in Plant Biology, 12(3), 259-266.

Hippler, F. W. R., Reis, I. M. S., Boaretto, R. M., Quaggio, J. A., \& Mattos Júnior, D. (2014). Características adsortivas de solos e o suprimento de zinco e manganês para os citros. Citrus Research \& Technology, 35(2), 73-83.

Hondebrink, M. A., Cammeraat, L. H., \& Cerdà, A. (2017). The impact of agricultural management on selected soil properties in citrus orchards in Eastern Spain: A comparison between conventional and organic citrus orchards with drip and flood irrigation. The Science of the Total Environment, 581-582(1), 153-160.

Instituto Nacional de Meteorologia - INMET. (2018). Banco de Dados Meteorológicos para Ensino e Pesquisa - BDMEP. Recuperado em 31 de junho de 2018, de http:// www.inmet.gov.br/portal/index.php?r=bdmep/bdmep/

Malavolta, E. (1976). Manual de química agrícola: nutrição de plantas e fertilidade do solo (527 pp.). Piracicaba: Editora Agronômica Ceres.

Mattos Junior, D., Quaggio, J. A., Cantarella, H., \& Alva, A. (2003). Nutrient content of biomass components of Hamlin sweet orange trees. Scientia Agrícola, 60(1), 155-160. 
Mattos Junior, D., Quaggio, J. A., Cantarella, H., \& Boaretto, R. M. (2009). Citros: manejo da fertilidade do solo para alta produtividade. Informações Agronômicas, 128(1), 5-12.

Minatel, A. L. G., Andrioli, I., Centurion, J. F., \& Natale, W. (2006). Efeitos da subsolagem e da adubação verde nas propriedades físicas do solo em pomar de citros. Engenharia Agrícola, 26(1), 86-95

Ministério da Agricultura Pecuária e Abastecimento MAPA. (2014). Manual de métodos analíticos oficiais para fertilizantes minerais, orgânicos, organominerais e corretivos (220 pp.). Brasília: MAPA/SDA/CGAL.

Nogueira, A. R. A., \& Souza, G. B. (2005). Manual de laboratórios: solo, água, nutrição vegetal, nutrição animal e alimentos (334 pp.). São Carlos: Embrapa Pecuária Sudeste.

Primavesi, A. (2014). Pergunte ao solo e às raízes: uma análise do solo tropical e mais de 70 casos resolvidos pela agroecologia (1. ed., 288 pp.). São Paulo: Nobel.

Primavesi, A. (2016). Manual do solo vivo - solo sadio, planta sadia, ser humano sadio (206 pp.). São Paulo: Expressão Popular.

Primavesi, O. (2012) Manejo ambiental agrícola para agricultura tropical agronômica e sociedade (840 pp.). Ouro Fino: Editora Ceres.

Quaggio, J. A., \& Raij, B. V. (1979). Comparação de métodos rápidos para a determinação da matéria orgânica em solos. Revista Brasileira de Ciência do Solo, 3(1), 184-187.

Raij, B. V. (1981). Avaliação da fertilidade do solo (142 pp.). Piracicaba: Instituto da Potassa \& Fosfato.
Raij, B. V. (2011). Fertilidade do solo e manejo de nutrientes (420 pp.). Piracicaba: International Plant Nutrition Institute.

Ribeiro, A. C., Guimarães, P. T., \& Alvarez, V. H. (1999).

Recomendações para o uso de corretivos e fertilizantes em Minas Gerais - 5a aproximação (359 pp.). Viçosa: Comissão de Fertilidade do Solo do Estado de Minas Gerais.

Richards, L. A. (1954). Diagnosis and improvement of saline and alkali soils (160 pp., Handbook, 60). Washington: U. S. Department of Agriculture.

Santos, E. S., Montenegro, A. A. A., Pedrosa, E. M. R., \& França, Ê. F. (2016). Crescimento e produção de repolho sob diferentes adubações na presença e ausência de cobertura morta em agricultura familiar. Irriga, 21(1), 74-89.

Silva, A. M. D. (2016). Adubação com potássio e boro sobre a produtividade, qualidade do fruto e teor de prolina em laranjeira 'Pêra' (Tese de doutorado). Universidade Federal do Amazonas, Manaus.

Sombra, K. E. S., Silva, A. C. C., Loureiro, F. L. C., \& Bastos, D. C. (2016). Citricultura desenvolvida na agricultura de base familiar do município de Russas, Ceará. Revista Cultura Agronômica, 25(3), 303-316.

Sombra, K. E. S., Silva, A. C. C., Loureiro, F. L. C., \& Uchôa, C. N. (2018). A citricultura como instrumento de preservação da agricultura familiar no semiárido cearense, Brasil. Revista de Extensão e Estudos Rurais, 7(1), 353-372.

Recebido: Julho 13, 2018 Aceito: Novembro 02, 2018

Como citar: Sombra K.E.S., Silva A.C.C., Loureiro F.L.C., Silva J.A.N, Santos Filho L.G., \& Uchôa C.N. (2018). Atributos Físicos e Químicos de Solos de Pomares de Laranja Doce no Semiárido do Ceará, Brasil . Citrus Research \& Technology, 39, e1041. https://doi.org/10.4322/crt.16318 